Chronic Obstructive Pulmonary Diseases: Journal of the COPD Foundation

\author{
Original Research
}

\title{
The Effect of Metabolic Syndrome Status on Lung Function and Patient-reported Outcomes in Patients with COPD Receiving Nebulized Glycopyrrolate
}

Brian Carlin, $\mathrm{MD}^{1}$ Gary T. Ferguson, $\mathrm{MD}^{2}$ Ayca Ozol-Godfrey, $\mathrm{PhD}^{3}$ Thomas Goodin, $\mathrm{PhD}^{3}$ Shahin Sanjar, $\mathrm{PhD}^{3}$

\begin{abstract}
Background: Concurrent chronic obstructive pulmonary disease (COPD) and metabolic syndrome (MetS) represent an important clinical phenotype with overlapping symptomology. The effect of MetS in COPD patients was assessed following treatment with nebulized glycopyrrolate (GLY; administered via eFlow ${ }^{\circledR} \mathrm{Closed}$ System Nebulizer).

Methods: Posthoc analyses were performed on pooled lung function, patient-reported outcome (PRO) and safety data by MetS status from patients treated with placebo, GLY 25 and 50 mcg twice daily in two 12-week studies (GOLDEN 3 and 4; N=1293). Patients with MetS were characterized as having $\geq 3$ of hypertension, hyperlipidemia, diabetes, body mass index $(\mathrm{BMI})>30 \mathrm{~kg} / \mathrm{m}^{2}$ risk factors. The results are presented for the Food and Drug Administration-approved GLY 25 mcg dose.

Results: A total of $25 \%$ of patients met MetS criteria. At baseline, the MetS subgroup had higher BMIs, more exsmokers, greater incidences of cardiovascular risk factors, and MetS-specific risk factors were 2-14 times higher than non-MetS. At 12 weeks, GLY produced significant, clinically important improvements (MetS: 0.121 L; nonMetS: $0.083 \mathrm{~L}$ ) in trough forced expiratory volume in 1 second. In the non-MetS group, significant improvements occurred in the St George's Respiratory Questionnaire (MetS: -2.28, p=0.157; non-MetS: -3.71) and Evaluating Respiratory Symptoms in COPD tool (MetS: 0.42, $p=0.574$; non-MetS: -1.61 ) total scores. Incidence of adverse events was similar with GLY versus placebo regardless of MetS status.

Conclusions: GLY was well-tolerated and significantly improved lung function regardless of MetS status, while significant PRO improvements occurred in non-MetS patients. These results highlight the importance of comorbidities on bronchodilator responses and patient symptoms in COPD patients.
\end{abstract}

Abbreviations: chronic obstructive pulmonary disease, COPD; metabolic syndrome, MetS; nebulized glycopyrrolate, GLY; patient-reported outcome, PRO; body mass index, BMI; cardiovascular disease, CV; twice daily, BID; closed system, CS; long-acting muscarinic antagonist, LAMA; U.S. Food and Drug Administration, FDA; long-acting beta2-agonist, LABA; Global initiative for chronic Obstructive Lung Disease, GOLD; forced expiratory volume in 1 second, FEV $\mathbf{1}$; forced vital capacity, FVC; St George's Respiratory Questionnaire, SGRQ; Evaluating Respiratory Symptoms in COPD tool, E-RS: COPD ${ }^{\circledR}$; adverse event, AE; serious adverse event, SAE; major adverse cardiovascular event, $\mathbf{M A C E}$; myocardial infarction, MI; intent-to-treat, ITT; inhaled corticosteroid, ICS; minimum, min.; treatment, tx; standard deviation, SD; least squares, LS; standard error, SE; confidence interval, CI; odds ratio, OR

Funding Support: The GOLDEN studies were supported by funding from Sunovion Pharmaceuticals, Inc. Medical writing support was funded by Sunovion Pharmaceuticals, Inc.

Date of Acceptance: April 22, 2020

Citation: Carlin B, Ferguson GT, Ozol-Godfrey A, Goodin T, Sanjar S. The effect of metabolic syndrome status on lung function and patientreported outcomes in patients with COPD receiving nebulized glycopyrrolate. Chronic Obstr Pulm Dis. 2020;7(4):315-326. doi: https:// doi.org/10.15326/jcopdf.7.4.2020.0145

1 Sleep Medicine and Lung Health Consultants, LLC, Pittsburgh, Pennsylvania
2 Pulmonary Research Institute of Southeast Michigan,

Farmington Hills, Michigan

3 Sunovion Pharmaceuticals, Inc., Marlborough, Massachusetts 


\section{Address correspondence to:}

Brian Carlin, MD

Sleep Medicine and Lung Health Consultants

P.O. Box 174

Ingomar, PA 15127 USA

Phone: + 1 (412) 351-6545

E-mail: bwcmd@yahoo.com

\section{Keywords:}

chronic obstructive pulmonary disease; COPD; eFlow; glycopyrrolate; metabolic syndrome; nebulized long-acting muscarinic antagonist; LAMA

\section{Note: The abstract of this paper was presented at the American Thoracic Society 2019 International Conference as a poster with interim findings.}

\section{Introduction}

Chronic obstructive pulmonary disease (COPD) is a progressive disease characterized by persistent respiratory symptoms and airflow limitation. ${ }^{1}$ Patients with COPD commonly present with coexisting comorbidities that impact quality of life and morbidity, such as cardiovascular (CV) disease, hypertension, and diabetes. ${ }^{2-4}$

Metabolic syndrome (MetS) is defined as a clustering of $\geq 3 \mathrm{CV}$ risk factors including hypertension, obesity, hyperlipidemia, and type 2 diabetes. ${ }^{5-7}$ There is a strong correlation between MetS and diminished airflow ${ }^{8}$ that has been shown to increase the risk of $\mathrm{CV}$ disease in patients with airway inflammation, impaired lung function ${ }^{9}$ and may worsen COPD progression. ${ }^{10}$ The reported prevalence of MetS in the general population, and in patients with COPD, varies by region. Global MetS prevalence ranges between approximately $20 \%$ and $50 \%, 8,11-14$ while prevalence of MetS in patients with COPD is similar or slightly higher compared with the general population, ranging between approximately $20 \%$ and $60 \%{ }^{8,10,13-22}$ Symptoms, such as shortness of breath (i.e., dyspnea), occur in patients with either COPD or CV disease, and may overlap in patients who have respiratory disease and CV comorbidities ${ }^{4,9}$ that could confound a diagnosis and initiation of appropriate therapy. ${ }^{4}$ This highlights the importance of the differential diagnosis and active management of COPD and concurrent comorbidities. ${ }^{18}$
Nebulized glycopyrrolate (GLY; LONHALA ${ }^{\circledR} 25$ mcg twice daily [BID]), delivered using the eFlow ${ }^{\circledR}$ Closed System (CS) Nebulizer (MAGNAIR ${ }^{\circledR}$; PARI Pharma GmbH; Starnberg, Germany) as a twice-daily, nebulized, long-acting muscarinic antagonist (LAMA) is approved by the U.S. Food and Drug Administration (FDA) for the long-term maintenance treatment of airflow obstruction in patients with COPD. ${ }^{23}$ This approval was based, in part, on the results from two 12-week, randomized, placebo-controlled, Phase 3 studies (GOLDEN 3 [NCT02347761] and GOLDEN 4 [NCT02347774]), which demonstrated that nebulized GLY treatment was well-tolerated and led to significant, clinically important improvements in lung function and patient-reported outcomes (PROs). ${ }^{24}$

A post-hoc analysis of pooled data from GOLDEN 3 and GOLDEN 4 was conducted to evaluate the effects of nebulized GLY 25 mcg BID on lung function, PROs and safety in COPD patients with and without MetS.

\section{Methods}

\section{Study Design and Treatment}

Study designs of GOLDEN 3 and GOLDEN 4 have been described previously ${ }^{24}$ and are shown in Figure 1. In brief, patients were randomized 1:1:1 and received placebo or GLY (25 or $50 \mathrm{mcg}$ BID), administered via the eFlow ${ }^{\circledR}$ CS Nebulizer. Randomization was stratified by CV risk (high/low) and background long-acting beta2-agonist (LABA) use (yes/no). The proportion of patients who continued background LABA use (with or without concomitant inhaled corticosteroids) during the treatment period was limited by protocol to approximately $30 \%$. Ipratropium bromide, as supplemental medication, and albuterol (salbutamol), as rescue medication, were permitted.

\section{Patients}

Detailed study entry criteria have been reported previously. ${ }^{24}$ Eligible patients were current or exsmokers, $\geq 40$ years of age with $\geq 10$ pack-year smoking history, with a clinical diagnosis of moderateto-very-severe COPD (defined using Global initiative for chronic Obstructive Lung Disease [GOLD] 2014 Report criteria), ${ }^{1}$ and with qualifying postbronchodilator (ipratropium $68 \mathrm{mcg}$ ) spirometry, including forced expiratory volume in 1 second $\left(\mathrm{FEV}_{1}\right) \leq 80 \%$ of predicted normal, $\mathrm{FEV}_{1}>0.7 \mathrm{~L}$, and 


\section{Figure 1. GOLDEN 3 and GOLDEN 4: 12-Week, Placebo-Controlled Studies ${ }^{9}$}

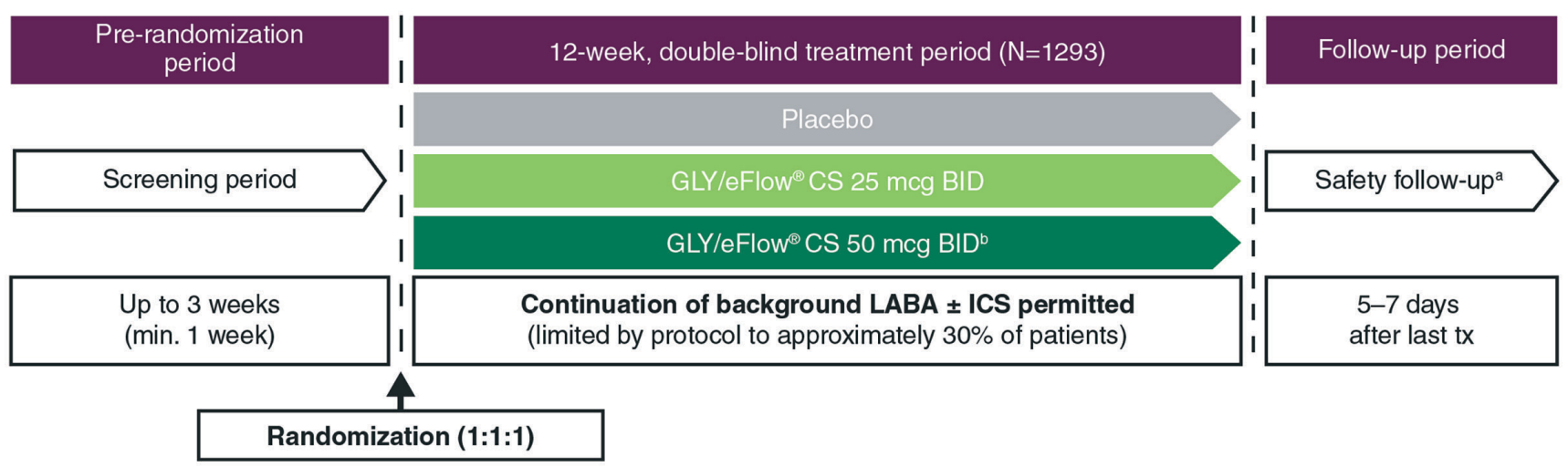

Serial spirometry was performed on Day 1 and at end of study and was only assessed in GOLDEN 3.

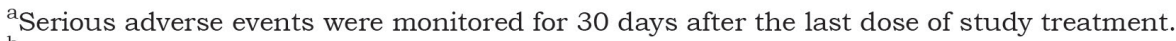

${ }^{\mathrm{b}}$ Data for the GLY $50 \mathrm{mcg}$ BID treatment arm are not presented in this post-hoc analysis but were included in the modelling.

GLY=nebulized glycopyrrolate; $\mathrm{CS}=$ closed system; BID=twice daily; min.=minimum; LABA=long-acting beta2-agonist; ICS=inhaled corticosteroid; tx=treatment

$\mathrm{FEV}_{1}$ /forced vital capacity $(\mathrm{FVC})$ ratio $<0.70$. Patients with severe comorbidities, history of or current unstable CV disease and/or long QT syndrome were excluded.

Study protocols were approved (GOLDEN 3: project approval number 28481; GOLDEN 4: project approval number 28482) by Quorum Review Institutional Review Board, North American (U.S. and Canadian) Board (Panel II) prior to patient enrollment, and were conducted in accordance with the protocols, International Council for Harmonization Good Clinical Practice guidelines, and Declaration of Helsinki. All patients provided written informed consent.

\section{Statistical Analyses}

The placebo, GLY 25 and $50 \mathrm{mcg}$ BID data from GOLDEN 3 and 4 were pooled ( $N=1293)$, and data for both GLY doses were included in all statistical models. Inclusion of the $50 \mathrm{mcg}$ BID data in the modelling does not confound the interpretation of the GLY 25 mcg BID dose. The data and results presented are for the FDA-approved GLY dose of 25 mcg BID.

Patients were grouped according to MetS status (MetS or non-MetS). MetS was defined as having at least 3 of the following: hypertension, hyperlipidemia, diabetes, or body mass index (BMI) $>30 \mathrm{~kg} / \mathrm{m}^{2}$. According to the protocol, high CV risk was determined based on a history of 1 or more of the following pre-specified disorders: ischemic heart disease, cerebrovascular disease, peripheral arterial disease, clinically significant arrhythmia (defined as any arrhythmia for which the patient was receiving or had received medication, or an interventional procedure, or identified by Holter monitoring at screening), heart failure, or hypertension.

Lung function and PRO endpoints included: change from baseline in trough $\mathrm{FEV}_{1}$, St George's Respiratory Questionnaire (SGRQ; including responders) total score, Evaluating Respiratory Symptoms in COPD tool (E-RS:COPD ${ }^{\circledR}$; including responders) total score, and rescue medication use.

Changes from baseline in trough $\mathrm{FEV}_{1}$ and E-RS:COPD total score were analyzed using a mixedmodel for repeated measures, and changes from baseline in SGRQ total score and rescue medication were analyzed by analysis of covariance. Minimum clinically important differences for the different measures were defined as: reduction in $S_{R R Q}{ }^{25}$ total score $\geq 4$; reduction in E-RS:COPD ${ }^{26}$ total score $\geq 2$. SGRQ responders were analyzed using logistic regression and E-RS:COPD responders were analyzed using a longitudinal logistic regression. All models included covariates for the baseline level of the appropriate outcome measure, CV risk, and background LABA use. All statistical procedures were performed using $\mathrm{SAS}^{\circledR}$ v9.2 or higher (SAS Institute Inc., Cary, North Carolina). Only data that were measured while on randomized blinded study treatment (i.e., on-treatment data) were analyzed. No adjustments were made for the post-hoc multiple comparisons. All $p$-value interpretations are made at 
the $5 \%$ significance level.

Safety outcomes included adverse events (AEs), serious AEs (SAEs), and major adverse CV events (MACEs; as determined by a blinded, independent committee, including CV death, non-fatal myocardial infarction [MI], and non-fatal stroke). Safety data were analyzed using descriptive statistics. AEs and SAEs were coded according to MedDRA v15.1.

Safety analyses were conducted using the safety population, and efficacy analyses using the intent-totreat (ITT) population, both consisting of all patients randomized to treatment who received $\geq 1$ dose of study drug.

\section{Results}

Patient Demographics and Baseline Characteristics Data for the GLY $25 \mathrm{mcg}$ BID and placebo treated patients ( $N=861)$ were reported by MetS ( $\mathrm{n}=217$ [25.2\%]; placebo: $n=116$; GLY 25 mcg BID: $n=101$ ) and non-MetS ( $n=644$ [74.8\%]; placebo: $n=314$; GLY 25 mcg BID: $n=330$ ) subgroups. The MetS subgroup included more males, ex-smokers, and patients with higher median BMI and weight than the non-MetS subgroup (Table 1). Baseline lung function, PRO total scores and rescue medication use were generally similar across the treatment groups and MetS/nonMetS subgroups. Most patients with MetS were classified as having high CV risk, based on a medical history of per protocol, pre-specified CV risk factors. Specifically, a substantially higher incidence of CV risk factors including hypertension and ischemia were present in MetS patients, whereas peripheral arterial disease and arrhythmias appeared to a similar extent in both MetS subgroups.

The incidence of MetS risk factors in the MetS versus non-MetS subgroup ranged between 2- (hypertension) and approximately14-times higher (diabetes) (Table 2 ). Over $90 \%$ of patients in the MetS subgroup had hypertension and/or hyperlipidemia, while less than $5 \%$ of patients in the non-MetS subgroup had diabetes.

\section{Efficacy}

Treatment with GLY 25 mcg BID resulted in significant $(p<0.001)$, clinically important improvements in placebo-adjusted trough $\mathrm{FEV}_{1}$ regardless of MetS status at week 12 (Figure 2). Additionally, GLY treatment resulted in significant placebo-adjusted improvements from baseline in SGRQ total score
(MetS: $p=0.157$; non-MetS: $p<0.001$; Figure 3A) and E-RS:COPD total score (MetS: $p=0.574$; non-MetS: $p<0.001$; Figure 3B) among non-MetS patients. The odds of being an SGRQ responder ( $\geq 4$-unit reduction in total score; Figure $4 \mathrm{~A}$ ) or an E-RS:COPD responder ( $\geq 2$-unit reduction in total score; Figure 4B) in the GLY 25 mcg BID treatment group were significant $(p<0.01)$ in the non-MetS subgroup.

Placebo-adjusted change from baseline in mean daily number of rescue medication puffs over 12 weeks was not significant for either subgroup (MetS: $p=0.881$; non-MetS: $p=0.154$; Figure 5).

\section{Safety}

Overall, GLY 25 mcg BID was well-tolerated in both MetS and non-MetS subgroups. A lower proportion of patients treated with GLY 25 mcg BID experienced AEs compared with placebo regardless of MetS status, and the incidences of any AE in MetS and non-MetS patients were similar (Table 3). The most common AEs across treatment groups were cough, worsening of COPD, and dyspnea, and were generally similar in MetS and non-MetS subgroups. Patients treated with GLY $25 \mathrm{mcg}$ BID in both the MetS and non-MetS subgroups experienced fewer SAEs than patients taking placebo.

The incidences of AEs related to cardiac disorders or hypertension in patients treated with GLY 25 mcg BID were similar between MetS and non-MetS subgroups and were lower compared with the corresponding placebo treatment groups. A MACE occurred only in 2 patients, both in the MetS subgroup taking placebo (both non-fatal MIs; incidence rate per thousand person-years $=16.4$ ).

\section{Discussion}

Clinically, patients with concurrent COPD and MetS present important challenges in disease management with bronchodilators based on overlapping symptomology. Despite the marked prevalence of comorbidities among patients with COPD, there have been limited studies assessing the impact of comorbidities on bronchodilator therapy. The results showed lung function improvements with GLY 25 mcg BID were consistent and significantly greater than placebo in both MetS and non-MetS subgroups; however, PROs were only significantly improved with GLY 25 mcg BID among patients in the non- 


\section{Table 1. Patient Demographics and Baseline Characteristics, by Metabolic Syndrome Subgroup (Safety Population)}

\begin{tabular}{|c|c|c|c|c|}
\hline \multirow[b]{2}{*}{ Parameter } & \multicolumn{2}{|c|}{ Mets } & \multicolumn{2}{|c|}{ Non-Mets } \\
\hline & $\begin{array}{c}\text { Placebo } \\
n=116\end{array}$ & $\underset{n=101}{\text { GLY } 25 \text { mcg BID }}$ & $\begin{array}{l}\text { Placebo } \\
n=314\end{array}$ & $\begin{array}{c}\text { GLY } 25 \mathrm{mcg} B I D \\
n=330\end{array}$ \\
\hline Age, years, median (range) & $67.0(46-84)$ & $66.0(46-81)$ & $63.0(41-84)$ & $63.0(40-83)$ \\
\hline Male, n (\%) & $72(62.1)$ & $63(62.4)$ & $163(51.9)$ & $179(54.2)$ \\
\hline BMI, $\mathrm{kg} / \mathrm{m}^{2}$, median (range) & $33.2(19.9-71.6)$ & $33.2(19.3-51.2)$ & $26.7(16.3-56.2)$ & $25.7(14.7-53.4)$ \\
\hline Weight, kg, median (range) & $98.9(45.0-226.9)$ & $98.2(63.3-155.6)$ & $77.0(44.8-164.6)$ & $76.4(41.2-153.3)$ \\
\hline Ex-smoker, n (\%) & $75(64.7)$ & $61(60.4)$ & 137 (43.6) & $130(39.4)$ \\
\hline $\mathbf{F E V}_{\mathbf{1}}, \mathrm{L}$, mean $(\mathrm{SD})$ & $\begin{array}{r}1.403(0.453) \\
{[n=113]}\end{array}$ & $\begin{array}{r}1.253(0.421) \\
{[\mathrm{n}=95]}\end{array}$ & $\begin{array}{r}1.297(0.490) \\
{[\mathrm{n}=302]}\end{array}$ & $\begin{array}{r}1.346(0.516) \\
{[\mathrm{n}=314]}\end{array}$ \\
\hline FEV $_{1} \%$ Predicted, ${ }^{a}$ mean (SD) & $54.2(12.0)$ & $50.2(12.5)$ & $51.4(14.1)$ & $52.4(14.6)$ \\
\hline $\mathbf{F E V}_{\mathbf{1}} / \mathbf{F V C},{ }^{\mathrm{a}} \%$, mean $(\mathrm{SD})$ & $57.4(9.1)$ & $56.1(8.8)$ & $53.6(9.9)$ & $53.8(10.3)$ \\
\hline SGRQ Total Score, median (range) & $\begin{array}{r}48.07(6.18-90.46) \\
{[n=110]}\end{array}$ & $\begin{array}{r}48.25(0.75-88.49) \\
{[\mathrm{n}=94]}\end{array}$ & $\begin{array}{r}47.06(1.13-98.21) \\
{[\mathrm{n}=300]}\end{array}$ & $\begin{array}{r}50.00(0-95.94) \\
{[\mathrm{n}=313]}\end{array}$ \\
\hline E-RS:COPD Total Score, median (range) & $\begin{array}{r}11.86(0-29.86) \\
{[n=113]}\end{array}$ & $\begin{array}{r}11.14(0-30.43) \\
{[\mathrm{n}=99]}\end{array}$ & $\begin{array}{r}11.71(0-34.71) \\
{[\mathrm{n}=307]}\end{array}$ & $\begin{array}{r}12.43(0-32.43) \\
{[\mathrm{n}=321]}\end{array}$ \\
\hline $\begin{array}{l}\text { Average Rescue Medication puffs per } \\
\text { day, median (range) }\end{array}$ & $\begin{array}{r}2.00(0-11.3) \\
{[n=107]}\end{array}$ & $\begin{array}{r}3.00(0-9.1) \\
{[\mathrm{n}=89]}\end{array}$ & $\begin{array}{r}2.57(0-14.1) \\
{[\mathrm{n}=282]}\end{array}$ & $\begin{array}{r}3.00(0-10.4) \\
{[n=303]}\end{array}$ \\
\hline High CV Risk, ${ }^{\mathrm{b}} \mathrm{n}(\%)$ & $111(95.7)$ & $96(95.0)$ & $167(53.2)$ & $179(54.2)$ \\
\hline Hypertension & $107(92.2)$ & $93(92.1)$ & $156(49.7)$ & $158(47.9)$ \\
\hline Ischemic Heart Disease & $20(17.2)$ & $23(22.8)$ & $27(8.6)$ & $30(9.1)$ \\
\hline Heart Failure & $10(8.6)$ & $2(2.0)$ & $4(1.3)$ & $9(2.7)$ \\
\hline Cerebrovascular Disease & $8(6.9)$ & $4(4.0)$ & $8(2.5)$ & $9(2.7)$ \\
\hline Peripheral Arterial Disease & $8(6.9)$ & $6(5.9)$ & $23(7.3)$ & $16(4.8)$ \\
\hline Clinically Significant Arrhythmia & $1(0.9)$ & $3(3.0)$ & $5(1.6)$ & $17(5.2)$ \\
\hline
\end{tabular}

aPost-bronchodilator (ipratropium bromide, $68 \mathrm{mcg}$ ).

${ }^{b}$ High CV risk was defined as a pre-existing diagnosis of hypertension, ischemic heart disease, heart failure, cerebrovascular disease, peripheral arterial disease, or clinically significant arrhythmia.

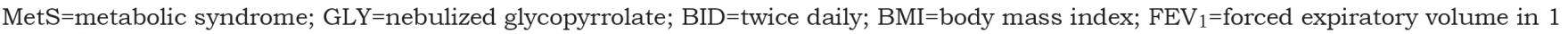
second; $\mathrm{SD}=$ standard deviation; $\mathrm{FVC}=$ forced vital capacity; SGRQ=St George's Respiratory Questionnaire; $\mathrm{E}-\mathrm{RS}$ : $\mathrm{COPD}=\mathrm{Evaluating}$ Respiratory Symptoms in COPD tool; $\mathrm{CV}=$ cardiovascular

MetS subgroup. Importantly, GLY 25 mcg BID was well-tolerated with similar AE incidence across MetS subgroups. While the GOLDEN 3 and 4 studies were not prospectively designed to assess the effect of MetS on COPD, the results of this post-hoc analysis provides valuable insights for clinicians to help guide the individualized care of complex COPD patients.

In GOLDEN 3 and GOLDEN 4, baseline lung function and SGRQ total scores were comparable to values reported in the literature for COPD patients with MetS. For patients with COPD and MetS, mean post-bronchodilator baseline $\mathrm{FEV}_{1} \%$ predicted in GOLDEN 3 and 4 (50.2\%-54.2\%) was similar to that reported across 5 studies (in a systematic review of 19 studies) by Cebron Lipovec et $\mathrm{al}^{15}(54 \%[\mathrm{n}=414])$ and in a prospective cohort analysis by Breyer et al ${ }^{16}(58 \%$ [n=101]). Mean baseline FEV $1 /$ FVC in COPD patients with MetS from GOLDEN 3 and 4 (56.1\%-57.4\%) was similar to mean values reported by Piazzolla et al ${ }^{17}$ (60\% [n=60]) and Park et $\mathrm{al}^{13}$ (56\% [n=54]), although Breyer et al ${ }^{16}$ reported a somewhat lower ratio of $44.6 \%$ $(\mathrm{n}=101)$. Breyer et $\mathrm{al}^{16}$ also reported a mean baseline SGRQ total score of 52.7 for COPD patients with MetS, which is consistent with the median baseline values in GOLDEN 3 and 4 (48.1-48.3).

Variability of placebo response, particularly in 
patients with MetS in the placebo treatment group, may have contributed to the effect sizes of outcomes in this post-hoc analysis. This could be due to the difference in patient numbers, as the non-MetS subgroup was

\section{Table 2. Metabolic Syndrome Cardiovascular Risk Factors (Safety Population)}

\begin{tabular}{l|c|c|c|c|}
\hline & \multicolumn{2}{|c}{ MetS } & \multicolumn{2}{c}{ Non-MetS } \\
\cline { 2 - 5 } Risk Factor, & Placebo & GLY & Placebo & GLY \\
$\mathrm{n}(\%)$ & $\mathrm{n}=116$ & $25 \mathrm{mcg}$ & $\mathrm{n}=314$ & $25 \mathrm{mcg}$ \\
& & BID & & BID \\
& & $\mathrm{n}=101$ & & $\mathrm{n}=330$ \\
\hline
\end{tabular}

\begin{tabular}{l|llll} 
Diabetes & $78(67.2)$ & $65(64.4)$ & $15(4.8)$ & $14(4.2)$
\end{tabular}

Hyperlipidemia 107 (92.2) 99 (98.0) 136 (43.3) 137 (41.5) Hypertension $^{\mathbf{a}} 113$ (97.4) 95 (94.1) 155 (49.4) 162 (49.1) BMI > $\mathbf{3 0 ~ \mathbf { ~ k g } / \mathbf { m } ^ { \mathbf { 2 } }} 88$ (75.9) 83 (82.2) 68 (21.7) 63 (19.1)

${ }^{a}$ Hypertension included: increased blood pressure, essential hypertension, hypertension, metabolic syndrome, hypertensive retinopathy.

MetS=metabolic syndrome; GLY=nebulized glycopyrrolate; $\mathrm{BID}=$ twice daily; $\mathrm{BMI}=$ body mass index approximately 3-times larger than the MetS subgroup, or due to the comorbidities, which may have affected the appearance and resolution of symptoms among patients receiving placebo. It is also possible that the variability in trough $\mathrm{FEV}_{1}$ results could be due to the relatively high baseline $\mathrm{FEV}_{1}$ in the placebo MetS subgroup (1.403 L) compared with other subgroups (1.253-1.346 L), however, differences in SGRQ and E-RS:COPD results cannot be similarly explained, as baseline values were comparable across MetS status and treatments.

Significant differences in placebo-adjusted PROs were noted only in the non-MetS subgroup. This may be due to patients without MetS having better PRO responses, as they do not experience overlapping symptoms of MetS and COPD (e.g., shortness of breath), thereby making it easier to identify COPD-related symptom improvements following bronchodilator therapy.

GLY 25 mcg BID was well-tolerated regardless of MetS status, with a lower incidence of AEs, SAEs, CVrelated AEs and MACEs compared with placebo. CV risk factors are more common in patients with MetS,

\section{Figure 2. Pooled Analysis of Change from Baseline in Trough Forced Expiratory Volume in 1 Second at 12 Weeks, by Metabolic Syndrome Subgroup (Intent-To-Treat Population)}

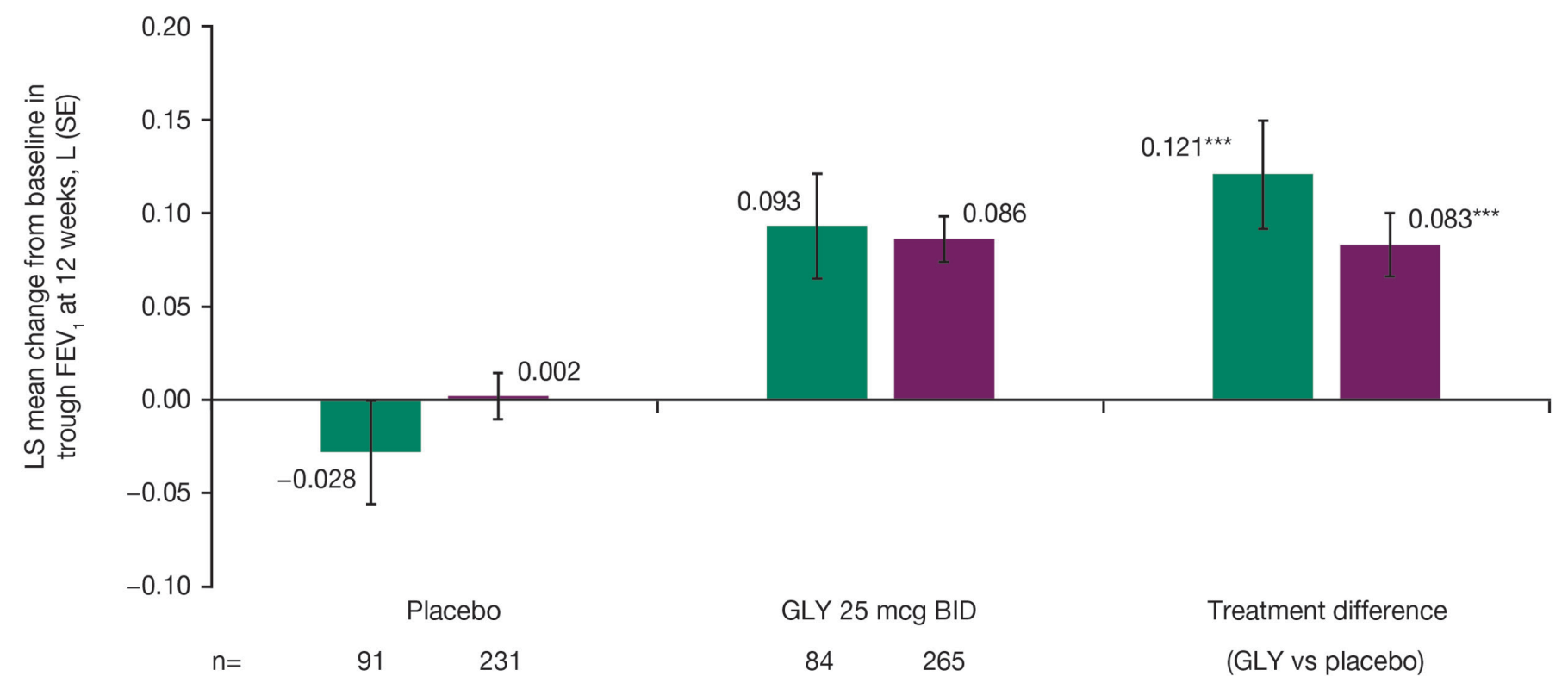

${ }^{* * *} p<0.001$ versus placebo

$\mathrm{LS}=$ least squares; $\mathrm{FEV}_{1}=$ forced expiratory volume in 1 second; $\mathrm{SE}=$ standard error; GLY=nebulized glycopyrrolate; BID=twice daily 


\section{Figure 3. Pooled Analysis of Change from Baseline in (A) St George's Respiratory Questionnaire Total Score, (B) Evaluating Respiratory Symptoms in COPD Total Score at 12 Weeks, by Metabolic Syndrome Subgroup (Intent-to-Treat Population)}

(A)

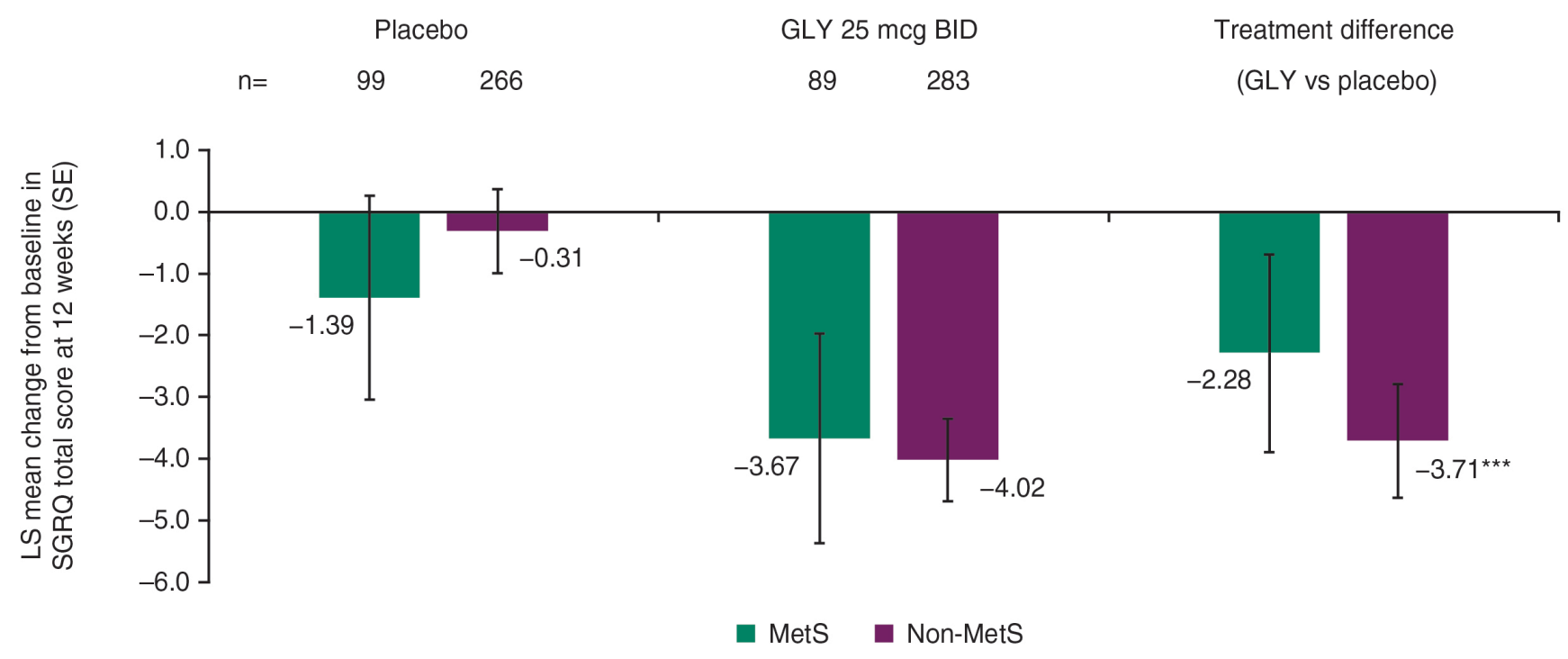

(B)

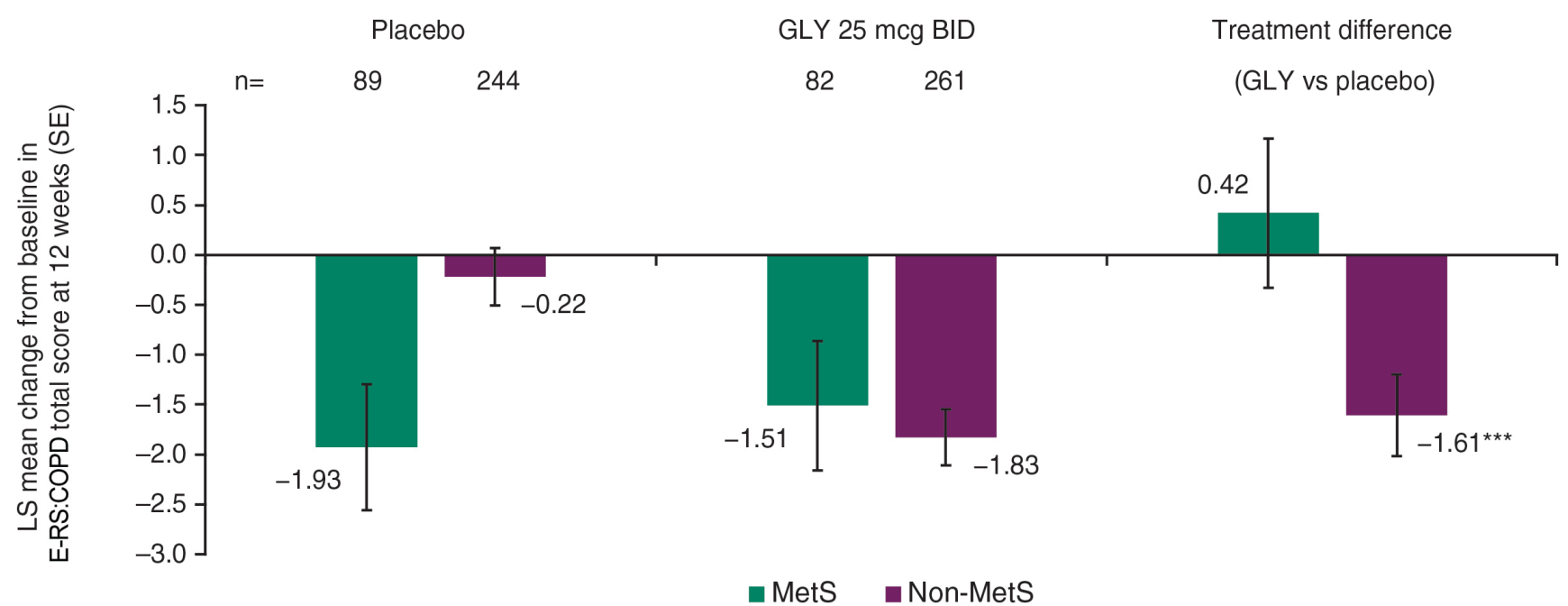

${ }^{* * *} p<0.001$ versus placebo

GLY=nebulized glycopyrrolate; BID=twice daily; LS=least squares; SGRQ=St George's Respiratory Questionnaire; SE=standard error; MetS=metabolic syndrome; E-RS:COPD=Evaluating Respiratory Symptoms in COPD

but there was no increase in the incidence of CVrelated AEs in patients treated with nebulized GLY in these studies, including those with MetS. These results support the overall and CV safety of GLY 25 mcg BID in patients with COPD, independent of MetS status.
Limitations to the current analysis include the posthoc patient classification, with MetS not included as a pre-specified sub-population involving the categorization of risk factors at screening but determined after study completion. Although patients 
Figure 4. Pooled Analysis of A) St George's Respiratory Questionnaire Responder Rates and B) Evaluating Respiratory Symptoms in COPD Responder Rates at 12 Weeks, by Metabolic Syndrome Subgroup (Intent-to-Treat Population)

(A)

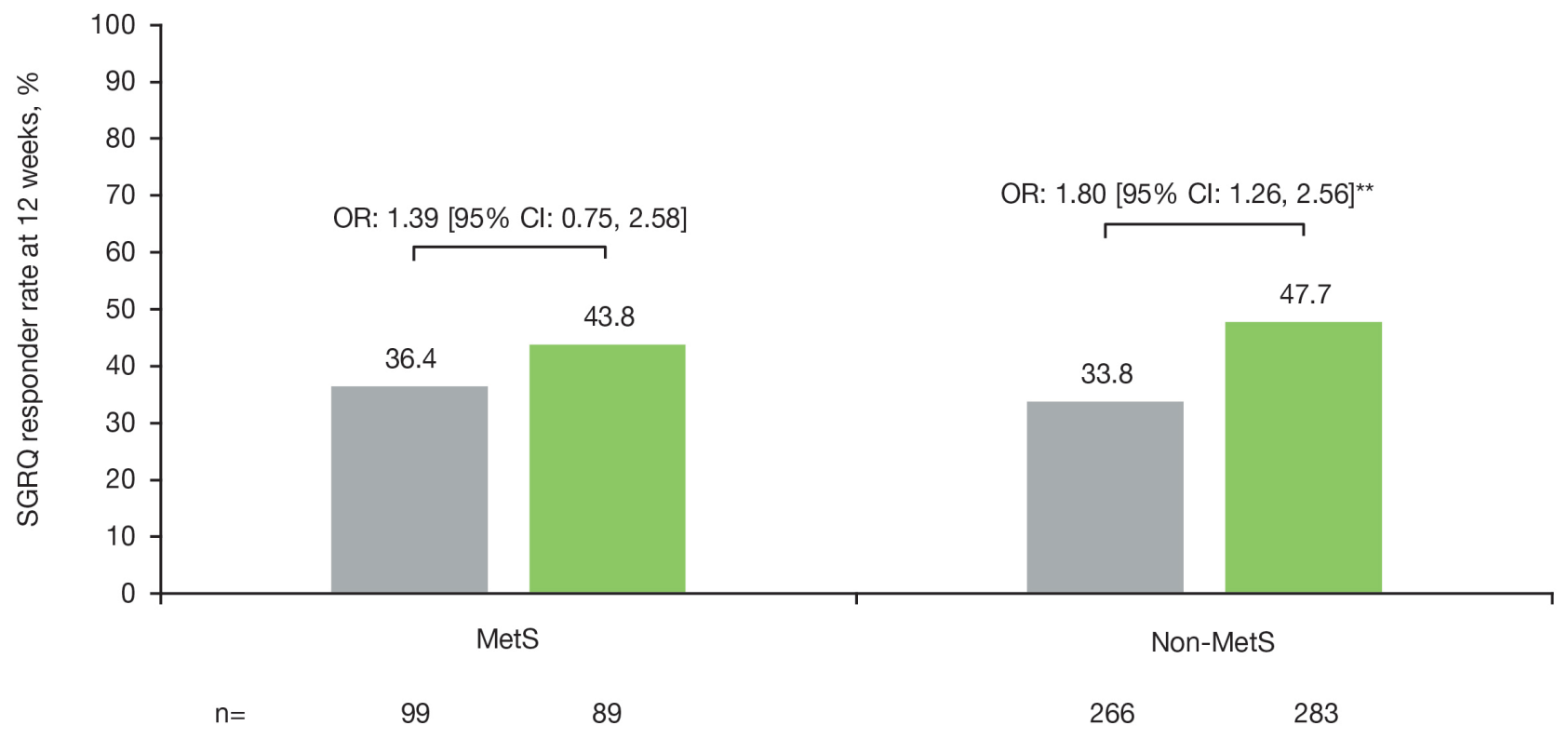

(B)

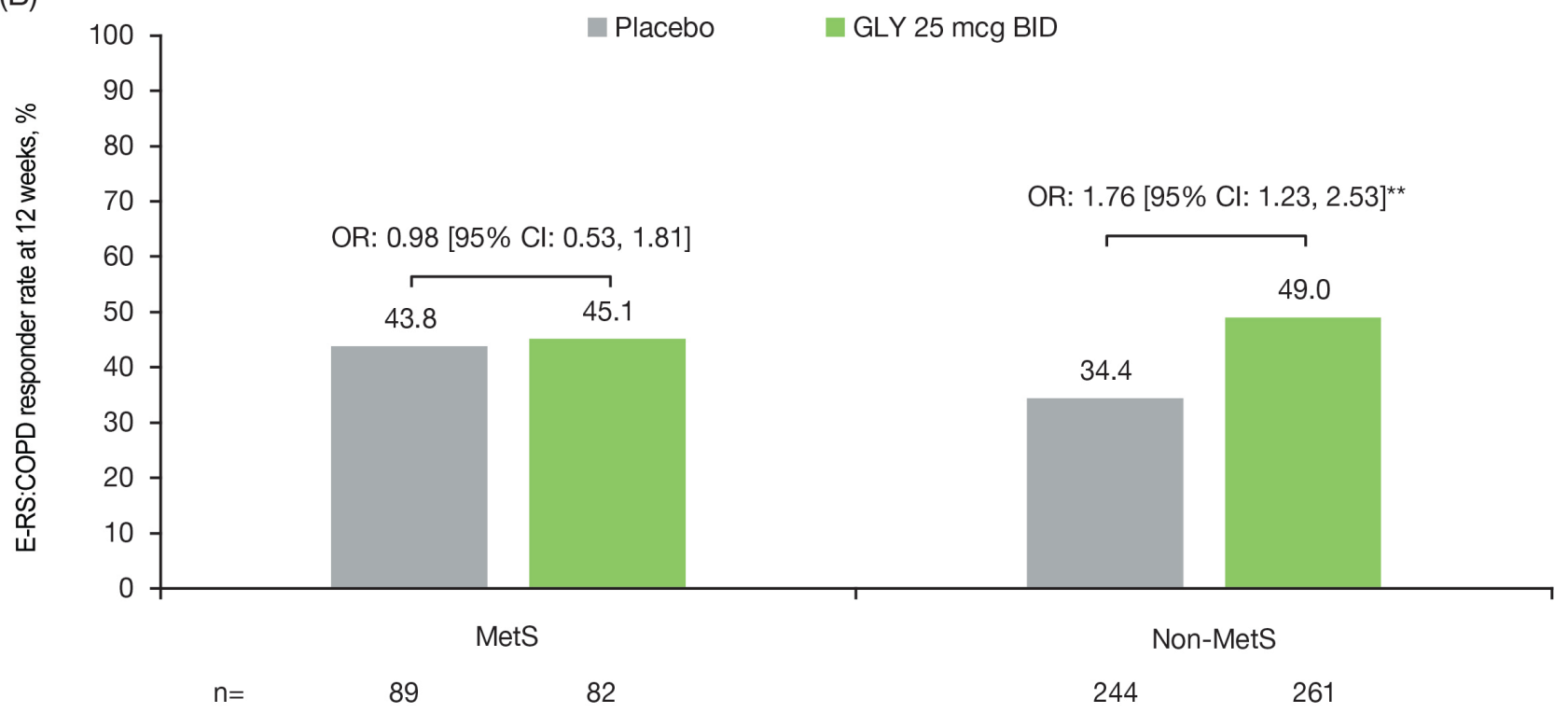

${ }^{* *} p<0.01$ versus placebo

SGRQ=St George's Respiratory Questionnaire; OR=odds ratio; CI=confidence interval; GLY=nebulized glycopyrrolate; BID=twice daily; MetS=metabolic syndrome; E-RS:COPD=Evaluating Respiratory Symptoms in COPD tool 


\section{Figure 5. Pooled Analysis of Change from Baseline in Mean Daily Number of Puffs of Rescue Medication Over 12 Weeks, by Metabolic Syndrome Subgroup (Intent-to-Treat Population)}

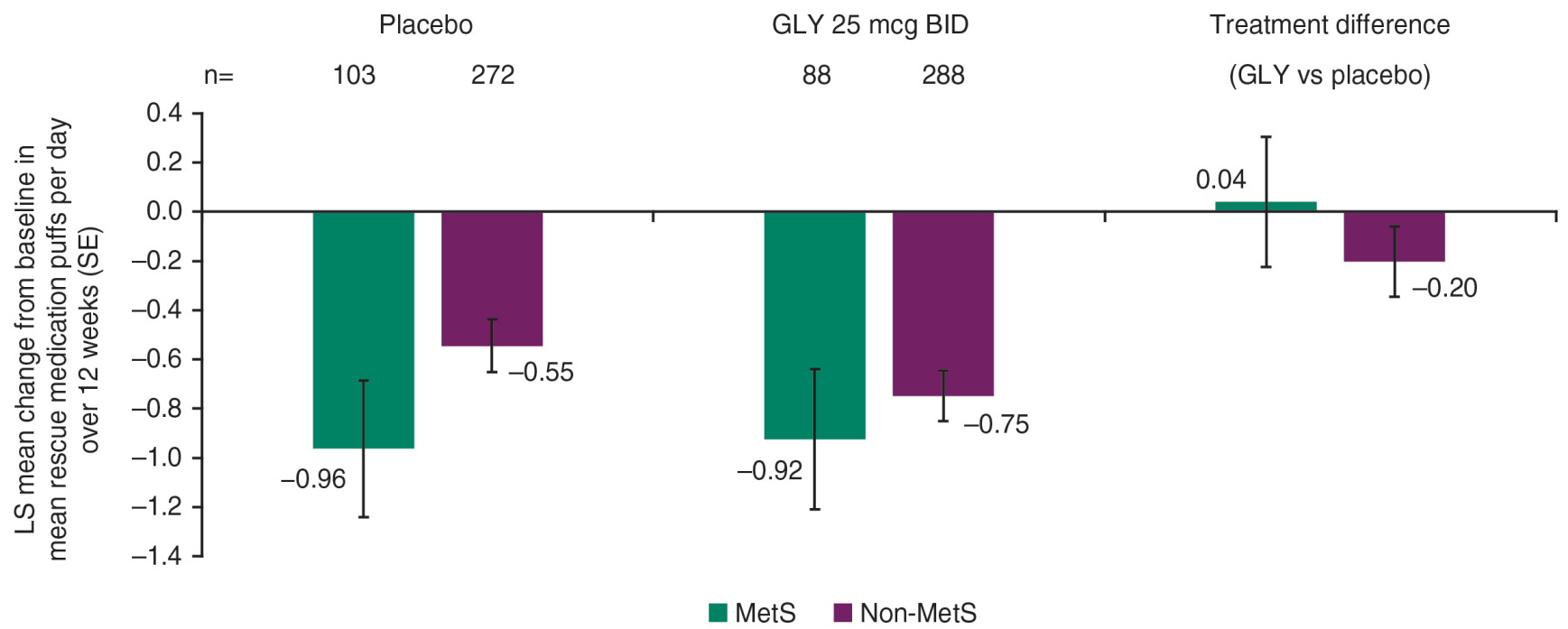

GLY=nebulized glycopyrrolate; BID=twice daily; LS=least squares; SE=standard error; MetS=metabolic syndrome

were stratified by CV risk, high risk only required one CV risk factor, rather than multiple factors as per MetS. This is evident in the large variation in the number of patients within each subgroup, even though the prevalence of MetS in COPD patients in this pooled population (25\%) is consistent with the lower end of reported prevalence (approximately 20\%-60\%), $8,10,13-$ 22 albeit the range of reported values is wide, across different geographic regions, and with varying population sizes.

\section{Conclusions}

Patients with COPD and concurrent MetS represent an important clinical phenotype with overlapping symptomology who may respond differentially to bronchodilator therapy. Treatment with GLY $25 \mathrm{mcg}$ BID produced lung function improvements compared with placebo, regardless of MetS status, whereas significant differences in placebo-adjusted PROs were noted only in the non-MetS subgroup. The incidence of AEs and SAEs were lower in patients treated with nebulized GLY compared with placebo in patients with and without MetS, including CV-related AEs. These results suggest that nebulized GLY $25 \mathrm{mcg}$ BID is a treatment option for patients with COPD, with or without concurrent MetS and highlights the importance of comorbidities on physiological and symptomatic responses to bronchodilators in COPD patients.

\section{Acknowledgments}

The authors would like to thank Shane Hornibrook from Sarepta Therapeutics, Inc., Diane Hall from Sunovion Pharmaceuticals, Inc., and Rajeshwari Sammishetty from Sage Therapeutics, Inc., for support with statistical analyses performed. Medical writing support was provided by Linda Townsend, PhD, and Hashem Dbouk, PhD, of Ashfield Healthcare, part of UDG Healthcare plc, and funded by Sunovion Pharmaceuticals, Inc.

\section{Author Contributions:}

All authors were involved in data analysis and interpretation, were involved at all stages of manuscript development, writing and revision, approved the final manuscript and agree to be held accountable for all aspects of the work. Gary T. Ferguson was involved in data acquisition.

Data Sharing Statement: Sunovion Pharmaceuticals, Inc., is part of a clinical trial data sharing consortium 


\section{Table 3. Summary of Adverse Events, ${ }^{a}$ Serious Adverse Events, and Cardiovascular-related Adverse Events, by Metabolic Syndrome Subgroup (Safety Population)}

\begin{tabular}{|c|c|c|c|c|}
\hline \multirow[b]{2}{*}{$\begin{array}{l}\text { Preferred Term, } \\
\text { n (\%) }\end{array}$} & \multicolumn{2}{|c|}{ MetS } & \multicolumn{2}{|c|}{ Non-MetS } \\
\hline & $\begin{array}{c}\text { Placebo } \\
n=116\end{array}$ & $\begin{array}{c}\text { GLY } \\
25 \text { mcg } \\
\text { BID } \\
n=101\end{array}$ & $\begin{array}{c}\text { Placebo } \\
n=314\end{array}$ & $\begin{array}{c}\text { GLY } \\
25 \mathrm{mcg} \\
\text { BID } \\
n=330\end{array}$ \\
\hline Any $\mathbf{A E}$ & $60(51.7)$ & $46(45.5)$ & 165 (52.5) & $141(42.7)$ \\
\hline Cough & $5(4.3)$ & $9(8.9)$ & $31(9.9)$ & $21(6.4)$ \\
\hline $\begin{array}{l}\text { COPD } \\
\text { Worsening }\end{array}$ & $5(4.3)$ & $7(6.9)$ & $32(10.2)$ & $21(6.4)$ \\
\hline Dyspnea & $2(1.7)$ & $3(3.0)$ & $11(3.5)$ & $18(5.5)$ \\
\hline $\begin{array}{l}\text { Urinary Tract } \\
\text { Infection }\end{array}$ & $2(1.7)$ & $4(4.0)$ & $4(1.3)$ & $5(1.5)$ \\
\hline Headache & 0 & $1(1.0)$ & $10(3.2)$ & $6(1.8)$ \\
\hline Any SAE & $8(6.9)$ & $5(5.0)$ & $16(5.1)$ & $8(2.4)$ \\
\hline CV-related AEs & & & & \\
\hline $\begin{array}{l}\text { Cardiac } \\
\text { Disorders }\end{array}$ & $5(4.3)$ & $1(1.0)$ & 5 (1.6) & $5(1.5)$ \\
\hline Hypertension & $3(2.6)$ & $2(2.0)$ & $4(1.3)$ & $3(0.9)$ \\
\hline
\end{tabular}

Including individual adverse events with incidence $\geq 3 \%$ in any treatment group

MetS=metabolic syndrome; GLY=nebulized glycopyrrolate; $\mathrm{BID}=$ twice daily; $\mathrm{AE}=$ adverse event; $\mathrm{COPD}=$ chronic obstructive pulmonary disease; $\mathrm{SAE}=$ serious adverse event; $\mathrm{CV}=$ cardiovascular that facilitates access for qualified researchers to selected anonymized clinical trial data. For up-todate information on data availability please visit https://www.clinicalstudydatarequest.com/StudySponsors.aspx and click on Sunovion.

\section{Declaration of Interest}

Dr. Brian Carlin has served on speakers' bureaus for Sunovion Pharmaceuticals Inc., and GlaxoSmithKline and is an advisory board member for Sunovion Pharmaceuticals Inc., GlaxoSmithKline, and Theravance. Dr. Gary T. Ferguson reports grants, personal fees, and non-financial support from Sunovion Pharmaceuticals Inc., during the conduct of the study; Dr Ferguson has also received grants, personal fees and non-financial support from AstraZeneca, Boehringer Ingelheim, Novartis, Pearl Therapeutics, Sunovion, Theravance, and GlaxoSmithKline; grants and personal fees from Verona and Sanofi; grants from Altavant; and personal fees from Mylan, Innoviva and Circassia. Dr. Ayca Ozol-Godfrey, Dr. Thomas Goodin, and Dr. Shahin Sanjar are employees of Sunovion Pharmaceuticals, Inc. 


\section{References}

1. Global Initiative for Chronic Obstructive Lung Disease (GOLD). Global strategy for the diagnosis, management and prevention of COPD, 2019. GOLD website. Published January 2019. Accessed June 3, 2019. http://goldcopd.org/

2. Divo M, Cote C, de Torres JP, et al. Comorbidities and risk of mortality in patients with chronic obstructive pulmonary disease. Am J Respir Crit Care Med. 2012;186(2):155-161. doi: https://doi.org/10.1164/rccm.201201-0034OC

3. Mannino DM, Thorn D, Swensen A, Holguin F. Prevalence and outcomes of diabetes, hypertension and cardiovascular disease in COPD. Eur Respir J. 2008;32(4):962.

doi: https://doi.org/10.1183/09031936.00012408

4. Cavaillès $A$, Brinchault-Rabin $G$, Dixmier $A$, et al. Comorbidities of COPD. Eur Respir Rev. 2013;22(130):454.

doi: https://doi.org/10.1183/09059180.00008612

5. Alberti KG, Zimmet P, Shaw J. Metabolic syndrome-a new world-wide definition. A consensus statement from the International Diabetes Federation. Diabet Med. 2006;23(5):469480. doi: https://doi.org/10.1111/j.1464-5491.2006.01858.x

6. International Diabetes Federation(IDF). The IDF consensus worldwide definition of the Metabolic Syndrome. IDF website. Published 2006. Updated July 29, 2020. Accessed September 5, 2019. https:/www.idf.org/e-library/consensus-statements/60idfconsensus-worldwide-definitionof-the-metabolic-syndrome. html

7. World Health Organization. Definition, diagnosis and classification of diabetes mellitus and its complications. WHO website. Published 1999. Accessed September 5, 2019. https:// apps.who.int/iris/bitstream/handle/10665/66040/WHO_ NCD_NCS_99.2.pdf

8. Lam KB, Jordan RE, Jiang CQ, et al. Airflow obstruction and metabolic syndrome: the Guangzhou Biobank Cohort Study. Eur Respir J. 2010;35(2):317-323.

doi: https://doi.org/10.1183/09031936.00024709

9. Lee HM, Zhao Y, Liu MA, et al. Impact of lung-function measures on cardiovascular disease events in older adults with metabolic syndrome and diabetes. Clin Cardiol. 2018;41(7):959-965.

doi: https://doi.org/10.1002/clc.22985

10. Chan SMH, Selemidis S, Bozinovski S, Vlahos R. Pathobiological mechanisms underlying metabolic syndrome (MetS) in chronic obstructive pulmonary disease (COPD): clinical significance and therapeutic strategies. Pharmacol Ther. 2019;198:160-188. doi: https://doi.org/10.1016/j.pharmthera.2019.02.013

11. Ranasinghe $\mathrm{P}$, Mathangasinghe $\mathrm{Y}$, Jayawardena $\mathrm{R}$, et al. Prevalence and trends of metabolic syndrome among adults in the Asia-Pacific region: a systematic review. BMC Public Health. 2017;17(1):101. doi: https://doi.org/10.1186/s12889-017-4041-1
12. Aguilar M, Bhuket T, Torres S, et al. Prevalence of the metabolic syndrome in the United States, 2003-2012. JAMA. 2015;313(19):1973-1974.

doi: https://doi.org/10.1001/jama.2015.4260

13. Park SK, Larson JL. Metabolic syndrome and associated factors in people with chronic obstructive pulmonary disease. West $J$ Nurs Res. 2014;36(5):620-642.

doi: https://doi.org/10.1177/0193945913512423

14. Marquis K, Maltais F, Duguay V, et al. The metabolic syndrome in patients with chronic obstructive pulmonary disease. $J$ Cardiopulm Rehabil. 2005;25(4):226-232; discussion 33-4. doi: https://doi.org/10.1097/00008483-200507000-00010

15. Cebron Lipovec N, Beijers RJ, van den Borst B, et al. The prevalence of metabolic syndrome in chronic obstructive pulmonary disease: a systematic review. COPD. 2016;13(3):399406. doi: https://doi.org/10.3109/15412555.2016.1140732

16. Breyer MK, Spruit MA, Hanson CK, et al. Prevalence of metabolic syndrome in COPD patients and its consequences. PLoS One. 2014;9(6):e98013.

doi: https://doi.org/10.1371/journal.pone.0098013

17. Piazzolla G, Castrovilli A, Liotino V, et al. Metabolic syndrome and chronic obstructive pulmonary disease (COPD): the interplay among smoking, insulin resistance and vitamin D. PLoS One. 2017;12(10):e0186708.

doi: https://doi.org/10.1371/journal.pone.0186708

18. Kim J, Yoo JY, Kim HS. Metabolic syndrome in South Korean patients with chronic obstructive pulmonary disease: a focus on gender differences. Asian Nurs Res. 2019;13(2):137-146. doi: https://doi.org/10.1016/j.anr.2019.03.002

19. Watz H, Waschki B, Kirsten A, et al. The metabolic syndrome in patients with chronic bronchitis and COPD: frequency and associated consequences for systemic inflammation and physical inactivity. Chest. 2009;136(4):1039-1046. doi: https://doi.org/10.1378/chest.09-0393

20. Ghanassia E, Jaussent A, Picot M-C, Préfaut C. Prévalence de l'insulinorésistance et du syndrome métabolique chez le sujet BPCO (French). Rev Mal Respir. 2006;23(4):393. doi: https://doi.org/10.1016/S0761-8425(06)71611-4

21. Ameen NM, El Deen Mohamed RS, El Mageed NIA, El Wahab MHA. The metabolic syndrome in patients with chronic obstructive pulmonary disease. Egypt $J$ Chest Dis Tuberc. 2016;65(3):593-596.

doi: https://doi.org/10.1016/j.ejcdt.2016.03.008

22. Minas M, Kostikas K, Papaioannou AI, et al. The association of metabolic syndrome with adipose tissue hormones and insulin resistance in patients with COPD without co-morbidities. COPD. 2011;8(6):414-420.

doi: https://doi.org/10.3109/15412555.2011.619600 
23. LONHALA MAGNAIR (glycopyrrolate) inhalation solution [package insert]. Sunovion Pharmaceuticals Inc.;2019.

24. Kerwin E, Donohue JF, Goodin T, et al. Efficacy and safety of glycopyrrolate/eFlow ${ }^{\circledR}$ CS (nebulized glycopyrrolate) in moderate-to-very-severe COPD: results from the glycopyrrolate for obstructive lung disease via electronic nebulizer (GOLDEN) 3 and 4 randomized controlled trials. Respir Med. 2017;132:238250. doi: https://doi.org/10.1016/j.rmed.2017.07.011

25. Jones PW. St George's Respiratory Questionnaire: MCID. COPD. 2005;2(1):75-79.

doi: https://doi.org/10.1081/COPD-200050513

26. Leidy NK, Murray LT, Monz BU, et al. Measuring respiratory symptoms of COPD: performance of the EXACT- Respiratory Symptoms Tool (E-RS) in three clinical trials. Respir Res. 2014;15:124. doi: https://oi.org/10.1186/s12931-014-0124-z 